\title{
Advanced Techniques of Bovine Semen Analysis
}

\author{
Mihai CENARIU ${ }^{1 *}$, Emoke PALL ${ }^{1}$, Mihai BORZAN² Liviu BOGDAN ${ }^{1}$ \\ and Ioan GROZA ${ }^{1}$ \\ ${ }^{1}$ Department of Animal Reproduction and Reproductive Pathology. University of Agricultural Sciences and \\ Veterinary Medicine Cluj-Napoca, Romania. \\ ${ }^{2}$ Department of Animal Breeding. University of Agricultural Sciences and Veterinary Medicine Cluj- \\ Napoca, Romania. \\ *corresponding author: mihai.cenariu@usamvcluj.ro
}

Bulletin UASVM Veterinary Medicine 75(1)/2018

Print ISSN 1843-5270; Electronic ISSN 1843-5378

doi:10.15835/buasvmcn-vm:004317

\begin{abstract}
The aim of the study was to assess bull semen fertility parameters using the classical techniques of sperm quality evaluation (density, motility, viability, and morphology, evaluated by light microscopy, in addition to concentration, evaluated via the hemocytometer and microspermatocrit), as well as advanced techniques, like computer assisted sperm analysis (CASA) and flow cytometry. Results obtained for classical techniques were comparable to those obtained by automated methods, without significant differences between parameters. The classical methods were inexpensive but required more time and attention, while the operator's experience was a key element for accurate assessment of sperm parameters. The advanced techniques were fast and objective, but required expensive equipment and dedicated personnel, with proper training in the field. Therefore, classical techniques are suitable for clinics where occasional evaluation of bulls' fertility parameters is performed, while the advanced methods should be implemented in semen companies, as well as in fertility clinics and research laboratories.
\end{abstract}

Keywords: bull semen, CASA, fertility, FACS, light microscopy

\section{Introduction}

The primary goal of sperm analysis, whether it may be via the classical or the novel methodology, is to inexpensively, rapidly, and above all else objectively and accurately predict the fertilization capacity of any given sample (Mann and LutwakMann, 2012; Amann and Waberski, 2014).

Classical methodology is indicated mainly through microscopic analysis. This type of methodology only has the ability to measure a small portion of spermatozoa within a population sample, it is of time consuming nature, and is highly subjective due to the human intervention, no matter the level of competence (Mortimer et al., 2015). In accordance to the previously stated concerns about the negative aspect of classical methodology, there is a generally recognized consensus that the classical methodology and techniques are not of a sufficient nature to describe and assess to the best of ability the biological sperm properties needed in order to ascertain fertility (Noakes et al., 2009; Singh et al., 2012).

Novel methodology is indicated mainly through the automated flow cytometry (FC) (Cordelli et al., 2005; Hossain et al., 2011) and through the computer assisted semen analysis (CASA) methods (Lu et al., 2014). Novel methodology has been implemented in order to objectively evaluate the spermatozoa of animals, to purposely predict the sample fertility, and to diagnose and specify the health of male reproductive organs. The animals in question are those destined for specific breeding and artificial insemination industries such as those dealing 
with the dairy industry or preservation of species foundations where objectivity and accuracy is key (Silva and Gadella, 2006).

The computer assisted semen analysis or the CASA method describes spermatozoa movements via specific motility parameters. These parameters are of a more detailed manner and again objectivity is of principle importance. In addition to the motility parameters, there is a classification of motile and immotile spermatozoa which is based on a well defined velocity formulation (Lu et al., 2014).

The second novel method is via the automated flow cytometry or FC. This can measure important sperm indications such as cell viability, acrosomal integrity and mitochondrial function, DNA structure and content. The FC method is an invaluable means to assess the spermatozoa cell quality. In a further simplification the method is ideal for an assessment of individual fertility potential (Nagy et al., 2003).

\section{Materials and methods}

The research was carried out on a total of 4 ejaculates, obtained from 4 Pinzgauer bulls (1 ejaculate from each male) commonly used for semen collection. Before collection, the bulls were assessed on their general state of well being and health via a general clinical examination, followed by an andrological examination. Semen was collected using an artificial vagina, while bulls mounted a cow in heats. Macroscopic examination of all ejaculates was performed, including volume, color, and aspect. Subsequently, ejaculates were placed at 37 degrees Celsius in order to avoid temperature shock. All semen samples were transported to the andrology laboratory within 2 hours of collection. Raw semen was analysed using classical methodology as well as novel techniques (CASA and flow cytometry).

\section{Classical Methods of Sperm Analysis}

1.1 Motility: One drop of semen was transferred onto a pre-warmed slide without a cover slip and examined via light microscopy. Under low magnification (4X objective), gross motility was observed. At this magnification individual sperm cannot be seen, but mass movement can in the form of a wave pattern or swirling. The classifications for this swirling pattern are as follows: Rapid Swirling - Very Good $(++++)$, Slower Swirling - Good (+++), Generalized
Oscillation - Fair (++), Sporadic Oscillation - Poor $(+)$, No Movement - Necrospermia (N).

Individual motility: one small drop of semen sample was examined using a $10 \mathrm{X}$ objective on a slide with a cover slip; avoiding air bubbles formation. A total of five fields were observed randomly in order to subjectively evaluate the number of progressively motile spermatozoa. In normal bulls, satisfactory progressive motility is greater than 30\% (Canadian Bovine Practitioners Association requires $60 \%$ motility for satisfactory classification) and greater than $90 \%$ progressive motility is an exceptional classification.

1.2 Density: Density of the semen sample was evaluated on the same slide as progressive motility. Three fields were viewed under the microscope. Interpretation was as follows:

- Dense Semen (D) - Distance between spermatozoa is smaller than spermatozoid head - one can subjectively assess that the ejaculate contains more than 1 billion spermatozoa/ml;

- Middle Sperm (M) - Distance between spermatozoa is approximately the same as the size of a spermatozoid head - the ejaculate contains $0.5-1$ billion spermatozoa $/ \mathrm{ml}$;

- Rare (R) - Distance between spermatozoa is bigger than the spermatozoid head - the ejaculate contains less than 0.5 billion spermatozoa $/ \mathrm{ml}$.

1.3 Concentration: In the assessment of concentration, two methods were performed:

Hemocytometer Method

In a Potain pipette up to the 0.5 division raw semen was aspirated. Next, a 3\% solution of $\mathrm{NaCl}$ was aspirated up to the 101 division. Then, the mixture was gently agitated and one droplet was loaded into the hemocytometer. The pipette tip was placed in the V-shaped groove on the hemocytometer in order to load the sample into the chamber $(\approx 15$ microliters). Through capillary action the fluid was drawn into the chamber. It was important not to overload the chamber, as doing so would skewer the results and give an inaccurate count. Additionally, the cover slip should also not be moved as to reduce to possibility of an inaccurate count.

The sample was allowed to settle for 2 to 3 minutes in order for the cells to stop drifiting around the chamber, and most will set in the same plane of focus. It was of utmost importance to not let the sample settle for too long or it would desiccate, concentrating the cells over the grid. 
The full grid on a hemocytometer contains nine squares and each one is $1 \mathrm{~mm}$ square. The central area for counting on the hemocytometer contains 25 large squares and each large square has 16 smaller squares. Priority is to those cells which are on the lines of two sides of the large square so as to avoid counting cells twice. A total of 5 squares from the 25 were counted and the resulting number was multiplied with $10^{6}$.

Concentration Via The Microspermatocrit

A capillary tube was filled with raw semen using a pipette. The capillary tube was filled up to two-thirds to three-quarters full. In one end of the tube clay was placed and filled as a plug. The tube was placed in the microhematocrit centrifuge, with the plugged end opposite to the center of the centrifuge. At the same time another tube was placed at the opposite end of the centrifuge as a counterbalance. The sample was centrifuged for 10 minutes at 14,000 RPM. The concentration was determined by reading the ratio of packed volume of cells as compared to the total volume of semen in the capillary tube, on a regular microhematocrit grid. One point on the grid corresponds to $200 \times 10^{6}$ spermatozoa/ml.

1.4 Morphology and Viability: The technique used was the nigrosin-eosin stain. The stain is effective, simple and allows sperm to be readily visualized. It is so called "live-dead" stain and allows assessing membrane integrity at the same time as morphology.

One drop of fresh sperm was put on the microscope slide using a pipette. A drop of the eosin stain was added to the semen sample followed by another drop of nigrosin. The sample was mixed and a smear was obtained using another microscopic slide.

The resulting slide was dried and placed under a bright light microscope for examination. The nigrosin stain produces a dark background onto which the sperm stand out as lightly colored. Normal live sperm exclude the eosin stain and appear white in color, unlike "dead" sperm which loose membrane integrity and therefor take up eosin further appearing pinkish or red in color. 200 spermatozoa were counted and the result was expressed as a percentage of viable sperm. Anomalies in the sperm were also investigated, searching for primary or secondary anomalies as well as immature spermatozoa, with a cytoplasmatic droplet still present.

\section{Novel Methods of Sperm Analysis}

2.1 Computer assisted sperm analysis (CASA): The research was performed using a Sperm Class Analyzer ${ }^{\circledR}$ CASA System. This system comprises of four modules: 1). Concentration and Motility; 2). Morphology; 3). Vitality; 4). Fragmentation, which are fully integrated and associated with an extensive database that will allow results and importation and exportation in many formats.

2.1.1. Motility and Concentration: For motility and concentration assessment, $1 \mu \mathrm{l}$ raw semen was diluted (1:300) with PBS (phosphate buffered saline) and placed on a pre-warmed microscopic slide and covered with a cover slip.

The microscope's green filter was inserted, Phase contrast 1 was selected, and the slide was inserted on the microscopic stage. The 10X negative phase contrast objective was selected for use. The image was focused in the microscope and then directed to the camera in order to see the image on the computer screen. The SCA Motility module was opened, Bull semen was selected from the parameter list, and the correct dilution ratio was entered. The Analyze button was pressed and 5 different fields were captured. Towards the end, the results button was pressed as well and the general results were displayed in the main window.

2.1.2 Sperm vitality: In sperm vitality assessment $10 \mu \mathrm{l}$ of raw semen was diluted (1:300) with PBS and placed in an empty vial and $1 \mu \mathrm{l}$ of BLUE eppendorf stain (Hoechst and trihydrochloride trihydrate) added, which was previously heated to 37 degrees Celsius. The vial was placed in a water bath at 37 degrees Celsius for five minutes. There after, $1 \mu \mathrm{l}$ of RED eppendorf stain was added (propidium iodide), which was previously heated to 37 degrees Celsius. A volume of $5-10 \mu \mathrm{l}$ of stained sample was placed on a standard slide with a cover slip and analyzed with CASA.

The specimen was placed onto the microscope stage, and the fluorescence module was started using the DAPI filter and the 20X objective. The image in the microscope field was focused and then directed towards the camera. The SCA Vitality module was opened and the proper configuration for Bull semen was selected. The Analyze button, was pressed, along with the image being focused in order to reach the best level of contrast so as to achieve a homogenous background similar to the 
Table 1. Macroscopic examination of raw semen in the four bulls

\begin{tabular}{ccccc}
\hline & Bull 1 & Bull 2 & Bull 3 & Bull 4 \\
\hline Volume & $8 \mathrm{ml}$ & $6 \mathrm{ml}$ & $7 \mathrm{ml}$ & $8 \mathrm{ml}$ \\
\hline Color & Creamy white & Creamy white & Creamy white & Creamy white \\
\hline Aspect & Dense & Dense & Dense & Dense \\
\hline
\end{tabular}

Table 2. Spermatic waves, motility and density results in the four bulls, using the classical techniques

\begin{tabular}{ccccc}
\hline & Bull 1 & Bull 2 & Bull 3 & Bull 4 \\
\hline Spermatic waves & ++++ & ++++ & ++++ & ++++ \\
\hline Motility & $100 \%$ & $95 \%$ & $90 \%$ & $95 \%$ \\
\hline Density & D & D & D & D \\
\hline
\end{tabular}

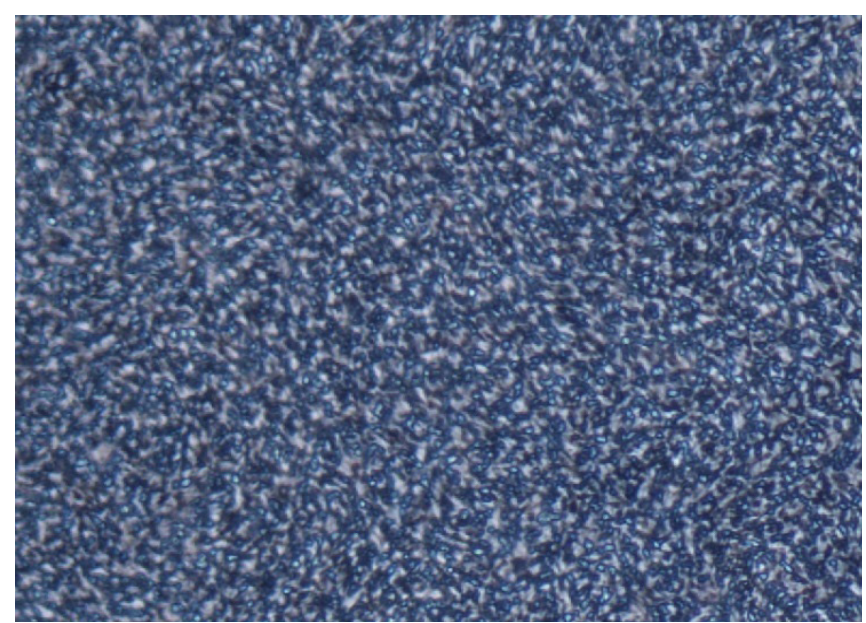

Figure 1. Microscopic image or raw bull semen (10x)

background color of the window. After the matter, 5 fields were captured, then the Results button was pressed and the resultant data was acquired.

\subsection{Flow cytometry}

2.2.1. Viability assesment by FACS analysis: In the experiments, sperm viability was assessed using the BD FACS Canto II flow cytometer using SYBR-14/PI staining. A sample of $10 \mu \mathrm{l}$ sperm was diluted (1:100) and $4 \mu$ l SYBR-14 as well as $1 \mu \mathrm{l}$ PI was added, agitated well and incubated for 20 minutes at 37 degrees Celsius. Examination was performed as soon as the incubation period ended.

The cytometer software was set up for obtaining information form the proper photodetectors (FL1-A for green and FL3-A for red). The photodetectors were set to "logarithmic" scale and to acquire "signal area". Since SYBR14 and PI spectra slightly overlap, fluorescence compensation was necessary. Samples were first analyzed for the FSC-A/ SSC-A signals, in order to identify the sperm population, gate out debris and electronic noise, and to assess the presence of excessive debris. A low flow pressure was used in order to read samples.

Unstained semen was used in the calibration of the BD FACS Canto II flow cytometer, followed by the analysis of stained samples. Fluorescence was detected using the $488 \mathrm{~nm}$, blue, air cooled, $20 \mathrm{~mW}$ solid state excitation laser, as well as the 530/30 filter for SYBR-14 and 575/26 filter for PI. The FACSDiva 6.1.2 software was used to perform the analysis.

\section{Results and discussions}

Macroscopic evaluation of raw semen obtained from all four bulls revealed appropriate characteristics of the ejaculates, while respecting all features of normal semen in bulls.

The volume of the ejaculates was between 6 and $8 \mathrm{ml}$, with a yellowish-white color and dense aspect (table 1, figures 1 and 2). The smell was also normal in all bulls, without any trace of urine odor. No foreign particles were noticed. 
Table 3. Concentration of spermatozoa in the four bulls by sperm count and microspermatocrit (billion spz. /ml)

\begin{tabular}{ccccc}
\hline & Bull 1 & Bull 2 & Bull 3 & Bull 4 \\
\hline Sperm count & 2.2 & 2.1 & 2.3 & 1.8 \\
\hline Microspermatocrit & 2.2 & 1.9 & 2.2 & 1.9 \\
\hline
\end{tabular}

Table 4. Viability of spermatozoa in the four bulls using eosin-nigrosine

\begin{tabular}{ccccc}
\hline & Bull 1 & Bull 2 & Bull 3 & Bull 4 \\
\hline $\begin{array}{c}\text { Viability (eosin- } \\
\text { nigrosine) }\end{array}$ & $98 \%$ & $96 \%$ & $92 \%$ & $97 \%$ \\
\hline
\end{tabular}

Table 5. CASA motility and concentration (billion spz. / $\mathrm{ml}$ ) results in the four bulls

\begin{tabular}{lcccc}
\hline & Bull 1 & Bull 2 & Bull 3 & Bull 4 \\
\hline CASA total motility & $100 \%$ & $98.75 \%$ & $90.41 \%$ & $98.31 \%$ \\
\hline $\begin{array}{c}\text { CASA progressive } \\
\text { motility }\end{array}$ & $90.27 \%$ & $58.11 \%$ & $52.84 \%$ & $59.64 \%$ \\
\hline CASA rapid velocity & $88.55 \%$ & $51.34 \%$ & $47.86 \%$ & $54.40 \%$ \\
\hline CASA Concentration & 2.176 & 2.089 & 2.311 & 1.879 \\
\hline
\end{tabular}

Table 6. Vitality of spermatozoa in the four bulls by CASA

\begin{tabular}{ccccc}
\hline & Bull 1 & Bull 2 & Bull 3 & Bull 4 \\
\hline Vitality (CASA) & $94.67 \%$ & $95.45 \%$ & $93.69 \%$ & $98.33 \%$ \\
\hline
\end{tabular}

Table 7. Viability of spermatozoa in the four bulls by flow cytometry

\begin{tabular}{ccccc}
\hline & Bull 1 & Bull 2 & Bull 3 & Bull 4 \\
\hline $\begin{array}{c}\text { Viability (flow- } \\
\text { cytometry) }\end{array}$ & $92.2 \%$ & $94.1 \%$ & $91.3 \%$ & $96.6 \%$ \\
\hline
\end{tabular}

Microscopic Evaluation of Bull Semen Using Classical Techniques

Raw semen obtained from the four bulls was evaluated using a light microscope in order to assess sperm waves, motility and density (Fig.1). For motility, the following results were obtained (Table 2):

When concentration was assessed using the two methods (sperm count using the Burker-Turk counting chamber and microspermatocrit), the following results were obtained (Table 3):

When viability was assessed using eosinnigrosine staining, the following results were obtained: (Table 4).

When morphological examination of spermatozoa was performed, on the same eosin-nigrosine stained smears, the percentage of primary and secondary abnormalities as well as immature spermatozoa was detected as being very low (between 5-10\%, which is acceptable for raw bull semen).

\section{Results Obtained for CASA Analysis}

When motility and concentration were assessed using the SCA Motility module of the CASA system, the following results were obtained (Table 5):

When sperm vitality was assessed by CASA, following results were obtained (Table 6):

When flow cytometry was used in order to assess sperm viability, following results were obtained (Table 7, Fig. 2):

Following the experiments regarding bull semen analysis using classical vs. modern techniques, several interesting aspects can be observed.

The classical methods using light microscopy were performed very fast, without the need of sophisticated equipment, but required a great deal of patience and meticulous work, performed by a specialized person, with lots of experience in the field. Most of the methods were very subjective and therefore an unexperienced worker could easily make important mistakes. 



Figure 2. Dot plots showing viability (FACS)

Motility, waves, as well as density were all assessed on a single unstained slide covered by a coverslip. Most importantly, motility is a vital parameter which can be easily and quickly assessed using this method, while the results were very similar to those obtained by CASA. Nevertheless, CASA was able to differentiate between progressively and non-progressively motile spermatozoa, which is extremely important, taking into consideration the fertilization capacity of those cells. As the results show very clearly, only one of the bulls (Bull 1) had great parameters regarding progressive motility, correlated with a proper velocity, thus providing an adequate level of confidence regarding its fertility. In the other three bulls, although gross motility was very good, the CASA analysis pointed out a large number of non-progressively motile spermatozoa, which have a questionable ability to fertilize an ovum. Moreover, if those spermatozoa were submitted to the process of cryopreservation, motility would decrease and their fertilization capacity would be even more unreliable.

It is often relatively easy to identify completely infertile bulls because their sperm usually are considerably less motile or have abnormal shapes. However, identifying subfertile bulls or individual subfertile semen collections is typically more difficult because the defects are not always very obvious. Sperm from subfertile bulls may appear normal, but insemination with this semen may produce lower conception rates. Identification of these subfertile bulls prior to being used heavily for AI would allow producers to reduce the number of cows bred to subfertile bulls and would increase reproductive efficiency. More accurate and sensitive measures of fertility are needed to identify these subfertile bulls (Miller, 1998).

Concentration is also one of the most important sperm parameters, which provides important information about a male's fertility, but also enables calculation of the amount of 
extender that needs to be added to raw semen in order to obtain standardized doses for artificial insemination (Bjorndahl, 2013). Assessment of concentration by sperm counting using the Burker-Turk hemocytometer was very difficult and time consuming, as all bulls taken into the experiment had a large number of spermatozoa (normal for large ruminants) which took a lot of patience and attention to count. This method can occasionally be performed, in the absence of other equipment, but is not suitable for laboratories that perform such tests on a regular basis. The microspermatocrit method was much easier to perform and the results were very reliable, as shown by CASA, which provided comparable results. The equipment needed is not expensive and can usually be found in any veterinary laboratory that also deals with blood samples.

Viability and morphology assessment by eosin-nigrosine stained smears provided reliable results, which were comparable with the CASA ones, but did also require examination and counting large numbers of spermatozoa, which was time consuming and difficult to perform. Acrosome integrity is also an extremely important parameter to be observed especially after cryopreservation (Aalseth and Saacke, 1985; Cross et al., 1986), which could not be assessed by classical techniques.

The computer assisted methods (CASA and flow cytometry) provided very close results in what viability of spermatozoa was concerned, which demonstrated their reliability and accuracy. These methods were very fast (results were basically generated within seconds) and the level of trust was very high, as there is no room for subjectivity or human error. The main disadvantage of those methods is that the equipment needed is very expensive and the personnel that operate it needs proper training.

Therefore, we recommend the use of classical semen analysis techniques in small clinics or under field conditions, when fertility of isolated males needs to be tested occasionally. In such cases, the investments needed to acquire expensive equipment are not sustained. On the contrary, in dedicated research laboratories or companies that process large volumes of semen daily, as well as in specialized clinics that deal with assisted reproductive technologies, such investments are absolutely necessary, as they provide quick, reproducible and very accurate results.

Using a CASA system as well as flow cytometry, the variation disappears, even between different laboratories and personnel. Furthermore, a CASA system permits to obtain verifiable data, as the images registered can be analyzed again, thus permitting an internal and external quality control, reproducibility and rapidity in obtaining quality data. All these advantages would allow a better male fertility diagnostic and consequently treatment, or decision making regarding the use of a particular male for reproduction (Moskovtsev et al., 2013).

\section{Conclusions}

The classical methods of sperm analysis were inexpensive but required more time and attention, while the operator's experience was a key element for accurate assessment of sperm parameters. The advanced techniques were fast and objective, but required expensive equipment and dedicated personnel, with proper training in the field. Therefore, classical techniques are suitable for clinics where occasional evaluation of bulls' fertility parameters is performed, while the advanced methods should be implemented in semen companies, as well as in fertility clinics and research laboratories.

Acknowledgments. This research did not receive any specific grant from funding agencies in the public, commercial, or not-for-profit sectors.

\section{References}

1. Aalseth EP, Saacke RG (1985). Morphological change of the acrosome on motile bovine spermatozoa due to storage at 4 degrees C. J Reproduction Fertility, 74: 473-478.

2. Amann RP, Waberski D (2014). Computer-Assisted Sperm Analysis (CASA): Capabilities and Potential Developments. Theriogenology, 81: 5-17.

3. Bjorndahl L (2013). Methods for sperm concentration determination. Springer Science \& Business Media LLC, (pp. 3-13).

4. Cordelli E, Eleuteri P, Leter G, Rescia M, Spano M (2005). Flow cytometry applications in the evaluation of sperm quality: semen analysis, sperm function and DNA integrity. Contraception, 72: 273-279.

5. Cross N, Morales P, Overstreet J, Hanson F (1986). Two simple methods for detecting acrosome-reacted humansperm, Gamete Res, 15: 213-226.

6. Hossain S, Johannisson A, Wallgren M, Nagy S, Pimenta Siqueira A, Rodriguez-Martinez H (2011). Flow cytometry 
for the assessment of animal sperm integrity and functionality: state of the art. Asian Journal of Andrology, 13: 406-419.

7. Lu JC, Huang YF, Lu NQ (2014). Computer-Aided Sperm Analysis: Past, Present, and Future. Andrologia, 46: 329338.

8. Mann TC, Lutwak-Mann (2012). Male Reproductive Function and Semen: Themes and Trends in Physiology, Biochemistry and Investigative Andrology. Springer Sciences \& Business Media, (pp. 25-69).

9. Miller DJ (1998). New Fertility Tests for Bulls. Dairy Cattle Illinois Livestock Trail http://livestocktrail.illinois.edu/ dairynet/paperDisplay.cfm?ContentID=172.

10. Mortimer ST, van der Horst G, Mortimer D (2015). The Future of Computer-Aided Sperm Analysis. Asian Journal of Andrology, 17: 545-553.
11. Moskovtsev SI, Clifford L, Librach (2013). Spermatogenesis Methods and Protocols. Springer Science \& Business Media LLC, (pp. 13-18).

12. Nagy S, Jansen J, Topper E, Gadella B (2003). A triplestain flow cytometric method to assess plasma-and acrosome-membrane integrity of cryopreserved bovine sperm immediately after thawing in presence of egg-yolk particles. Biology of Reproduction, 68: 1828-1835.

13. Noakes DE, Parkinson TJ, England GCW (2009). Veterinary Reproduction and Obstetrics. (9th ed). Saunders Elsevier.

14. Silva P, Gadella B (2006). Detection of damage in mammalian sperm cells. Theriogenology, 65: 958-978.

15. Singh A, Udai VS, Teotia SS (2012). Semen Evaluation in Farm Animals. DHR International Journal of Biomedical and Life Sciences, 2: 35-40. 